\title{
Native human dermis versus human acellular dermal matrix: A comparison of
}

\section{biaxial mechanical properties}

Mara Terzini $^{1}$, Alessandra Aldieri ${ }^{1}$, Elisabetta M. Zanetti ${ }^{2}$, Diana Massai ${ }^{1}$, Alberto L. Audenino ${ }^{1}$, and Cristina Bignardi ${ }^{1}$

1. Department of Mechanical and Aerospace Engineering, PolitoBIOMed Lab, Politecnico di Torino, Torino, Italy

2. Department of Engineering, University of Perugia, Perugia, Italy

\section{RESEARCH}

Please cite this paper as: Terzini M, Aldieri A, Zanetti EM, Audenino AL, Bignardi C. Native human dermis versus human acellular dermal matrix: A comparison of biaxial mechanical properties. AMJ 2018;11(8):434-442.

https://doi.org/10.21767/AMJ.2018.3487

\section{Corresponding Author:}

Mara Terzini

Department of Mechanical and Aerospace Engineering, Politecnico di Torino, C.so Duca degli Abruzzi 24, 10129 Torino, Italy

Email: mara.terzini@polito.it

\section{ABSTRACT}

\section{Background}

Human Acellular Dermal Matrices (HADMs), thanks to its mechanical resistance and it's not immunogenic response, is used in reconstructive surgery such as breast reconstruction procedures and hernia repairs. There is the need to investigate the mechanical response of HADMs when subjected to in vivo-like stresses.

\section{Aims}

In order to supply additional guidance to surgeons, in this work equi-biaxial experimental curves of native and decellularized human dermis are presented because it is essential to investigate the engineered tissue response when subjected to stresses comparable to those that occur in vivo.

\section{Methods}

HADMs specimens were biaxially characterized exploiting a customized biaxial conversion device entirely realized through rapid prototyping methods, and the HADM response to mechanical stimuli comparable to the in vivo deformation state was explored. From the derived data, stress-strain curves were evaluated, and the elastic moduli were extracted from the curve toe-region. As an indication of the fibre rearrangement rate, the slope of the stressstrain curve at higher strains was evaluated in a semi-log plane.

\section{Results}

The mean elastic modulus at low strains for the mediolateral direction (along Langer lines) resulted from 35 per cent to 87 per cent higher than the cranio-caudal one. Furthermore, medio-lateral specimens show lower rearrangement velocities at higher strains. Considering both directions, the decellularization process leads to a deterioration of the mechanical properties of the matrix.

\section{Conclusion}

The tested HADMs maintained the typical anisotropic dermis behaviour, dependent on the collagen network predominantly oriented along the Langer lines. Moreover, comparisons among HADMs and the native human reticular dermis demonstrated the mechanical strength loss at lower strains caused by the decellularization process.

\section{Key Words}

Human dermis, HADM, biaxial characterization, biaxial conversion device, rapid prototyping

\section{What this study adds:}

\section{What is known about this subject?}

Human Acellular Dermal Matrices (HADMs), derived from the human dermis decellularization, are highly anisotropic materials, and their mechanical response is strictly related to the native collagen network orientation with respect to Langer's lines. 


\section{What new information is offered in this study?}

This study introduced a custom made 3D printed conversion device for a uniaxial testing machine, which allows for the equi-biaxial testing of human dermis and HADMs. Stressstrain curves and descriptive parameters (elastic moduli at low strains and stress-strain curve slope at high strains) were measured and presented for native human dermis and HADMs.

\section{What are the implications for research, policy, or practice?}

The pre-operative planning of surgical reconstructions can take benefit from a deeper knowledge of the HADMs mechanical response under in-vivo like deformations and of the preferable graft orientation with respect to the surrounding host tissue.

\section{Background}

HADMs (Human Acellular Dermal Matrices) are generally used in skin replacement for burn patients, but as the understating of the dermis characteristics go on the indications for its use expand. The acellular dermal grafts were originally developed in the treatment of full-thickness burns, because of their not immunogenic response, handling, long shelf-life and expedite healing. They recently found application also in the repair of the oral mucosal. ${ }^{1}$ As well as in the skin coverage, in this ambit the HADMs use involves the same mechanism of incorporation and the same uncritical requisites in terms of mechanical response. The use of HADMs in load bearing applications began with the breast reconstructions ${ }^{2,3}$ and the hernia repairs, ${ }^{4}$ where the strength of the repair is mandatory to a successful outcome. Similar requisite is desired in applications such as the repair of rotator cuff tears, ${ }^{5-8}$ and the Achilles tendon augmentation, ${ }^{9,10}$ where HADMs are used as augmentation grafts, which facilitate the end-to-end anastomosis.

The biomechanical response of HADMs, in relation to the intended use, may be of clinical significance, especially in potentially load bearing applications. ${ }^{11}$ Because of its oriented fibrous structure, human dermis always exhibits a pronounced mechanical anisotropy. ${ }^{12,13}$ In order to fully characterize the HADM properties it can be performed a planar biaxial test that allows for a two-dimensional stressstate characterization of the tissue. Biaxial testing devices are much more elaborate then uniaxial ones because of numerous additional requirements (e.g., two loading directions, two load signals recording, optical measure of strain). Moreover, biaxial experiments on soft biological tissues are generally problematic, presenting unique challenges such as the small specimen size, the effects of different gripping techniques, the large specimen-tospecimen variability and the homogeneity of strain within the specimen. For instance, ensuring a firm specimen gripping without damaging the tissue is mandatory and challenging because of the above mentioned small specimen size, texture and humidity conditions. The preponderant solutions make use of thin threads (suture material), ${ }^{14-16}$ clips $^{17-19}$ and hooks. ${ }^{20,21}$ Besides is fundamental a non-contact strain measurement in order to avoid any mechanical interference with the specimen.

The strain optical measure is generally performed in a central target region which must be small enough and far from the outer edges to avoid the gripping effects. ${ }^{22}$ In this central region, the stress-strain field can be considered homogenous. $^{23}$ This assumption is based on the SaintVenant's principle which states that the stress distribution may be assumed to be independent from load application way, except in the immediate vicinity of where the load is applied. ${ }^{14}$ Nevertheless, the effects of load applications may be very different, depending on the gripping method: by clamp or by suture. The suture can possibly generate a discontinuous load transfer, also dependent on the number and closeness of the suture points. On the contrary, clamps would generate a continuous load transfer, but tested tissue could seem stiffer and slipping during the test can take place. In the clinical practice the implant of an allograft in vivo in the ambit of a reconstructive surgery takes place via suture, and therefore the application of the load in the immediate postoperative period will be punctual.

The first investigators that developed and used planar biaxial testing for soft biological tissues were Lanir et al. ${ }^{24,25}$ They investigated the mechanical properties of rabbit skin demonstrating the nonlinear and orthotropic stress-strain response of this tissue and the dependence of the latter on the specimen anatomic orientation. Moreover, they developed the most broadly used constitutive model for the biaxial response of skin. ${ }^{26}$ The biaxial characterization of soft tissues involved then extensively the cardiac and artery tissues, with the aim of implement constitutive models useful to carry out preclinical tests of intravascular devices, ${ }^{16-18,20,27}$ and the porcine meniscal. ${ }^{21}$ In many cases, the research outcomes are models' parameters obtained through the fitting of a mean curve. ${ }^{18,20}$ The assumption is that although the anisotropy of the tissue is obvious and confirmed by the biaxial tests, the response of the tissue along two orthogonal axes has no significant difference at physiological loadings. This assumption may not be true for the skin, which can reach high strains both in vivo and in 
vitro. It appears then remarkable the lack of published research on the biaxial properties of human dermis.

The aim of this work is to fill this lack showing equi-biaxial experimental curves of native and decellularized human dermis. In fact, not only a mechanical characterization aimed to optimize the chemical treatments required to obtain an engineered acellular matrix, ${ }^{28-30}$ but also the subsequent characterizations aimed at the investigation of the engineered tissue response when subjected to stresses comparable to those in vivo are essential to provide additional guidance to surgeons.

\section{Method}

\section{The biaxial conversion device}

As an accessory of a universal testing machine (a uniaxial Bose Electroforce ${ }^{\circledR}$ 3200), a customized biaxial testing device was designed, taking advantage of the machine own actuation and data acquisition systems and integrating the device with the missing sensors. Indeed, the load cell embedded in the testing machine was transferred into the conversion device (Figure 1(1)), and a further load cell was positioned along the orthogonal direction (Figure 1(2)). Each device component was realized through rapid prototyping methods (3D printer Stratasys uPrint SE Plus - Stratasys Inc., Edina, Minnesota, United States). The choice to take advantage of this technique greatly reduced materials and machining costs, yet forcing the device design. In fact, 3D printing machining is binding owing to: limited maximum size of the component, difficulties in realizing undercuts and cavities. Therefore, all the components have been conceived as multi-part assemblies, devising when needed alternative couplings between parts. The action of four commercial linear guideways (Hiwin ${ }^{\circledR}$ MGN) at an angle of $45^{\circ}$ with respect to the actuator axis allowed for the motion conversion from uniaxial to equi-biaxial, according to this operating principle: the vertical movement of the uniaxial tensile machine crosshead engenders the translation of the conversion device upper crosshead (Figure 1(3)) with a velocity "v", whereas the lower crosshead (Figure 1(4)) remains motionless. Simultaneously, the lateral links (Figure $1(5,6))$ move bottom-up with a " $/ 2$ " velocity and translate in the horizontally with a velocity of " $v / 2$ ", thus obtaining an equi-biaxial displacement. Due to the Bose Electroforce ${ }^{\circledR}$ 3200 maximum available stroke $(12.5 \mathrm{~mm})$, the maximum displacement of the moving crosshead engenders an identical $12.5 \mathrm{~mm}$ stroke in the orthogonal direction. Specimens are mounted on the biaxial testing device inserting 12 hooks evenly distributed on the four sides of the specimen at a $5 \mathrm{~mm}$ distance from the edges.
The handling of a biological sample which, by its nature, appears flabby and slippery is always laborious. These complications are magnified when a fine positioning is required, as needed in the case of biaxial characterization. Especially when performed by hooks and wires, the quality of the specimen gripping affects the homogeneity of the stress and strain field, since an uneven positioning of the load application points generates unbalanced boundary conditions which would lead to a reversal of the assumption of equi-biaxiality. This is the reason why a custom made accessory kit for the simple and repeatable positioning of the biological specimen was devised and realized through $3 D$ printing. Before actual use on biological tissues, the device functionality was tested. First of all, a highly unbalanced load state was verified, imposing a constant displacement rate of $0.16 \mathrm{~mm} / \mathrm{s}$ on a rubber specimen mounted within the fixture first in a horizontal configuration leaving free the vertical hooks, and then, in the opposite configuration. A Canon EOS 5D Mark II digital camera was used for the recording of each test and grips displacement was tracked post-processing the video recordings. The obtained strain-rates in the vertical and horizontal directions differs from the nominal imposed speed of about 1 per cent, confirming the grips motion reliability with unbalanced loads. Moreover, a local strain analysis was performed on an isotropic rubber specimen, to verify the homogeneity of the strain field in the central area of the specimen. A Matlab-based Digital Image Correlation (DIC) code, implemented at the University of Illinois in 2013, ${ }^{31}$ was used to track a random pattern of speckles drawn on the specimen surface. Figure 2 shows the digital image correlation results, in terms of strain distribution along the $x$ and $y$ directions, and Von Mises equivalent strain distribution. Two different time instants are highlighted: an intermediate (30 s) and a final (60 s) one. The equi-biaxiality of the strain distribution is confirmed by the similar behaviour along the two orthogonal directions. From Figure 2 an unexpected distribution at the corners of the specimen can be noted, which is expressed in higher values of deformation. This is induced by the out of plane movement of the not-constrained corners. This would not happen with the use of a cruciform specimen, considered as a standard shape for biaxial material characterizations. Despite this, the device was designed for the gripping of biological tissues notoriously difficult to handle and cut into complex shapes. The square shape is the most frequently adopted solution for the biaxial characterization of biological tissues. $^{15,17,18,20}$ 
Figure 1: Customized biaxial conversion device: the long black arrow is the vertical velocity $v$ of the moving crosshead; the short black arrows are the vertical velocities $v / 2$ of the lateral links; the white arrows are the horizontal velocities $v / 2$ of the lateral links

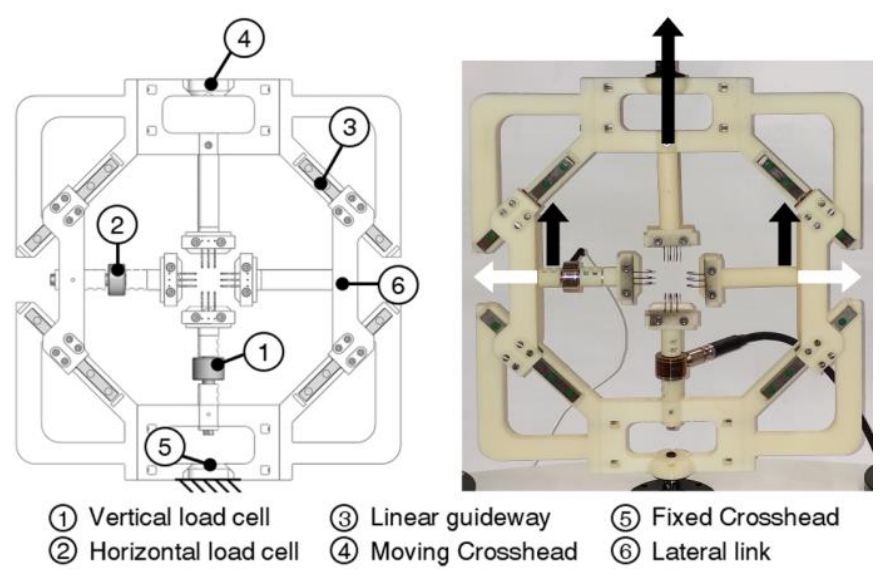

\section{Samples}

Dermis strips unfit for transplantation were used for this research after the approval of the Institutional Ethical Board of Azienda Ospedaliera Universitaria Città della Salute e della Scienza of Turin, Italy, (approved on January 23rd, 2012 with protocol number 0006730), and written informed consent was obtained from all study participants. Large strips of dermis tissue, collected from the backs of three human donors ( $A, B$ and $C$ ), were dissected along the craniocaudal direction. They were then decellularized using incubation in DMEM medium for five weeks. The treatment type and its duration were set according to the results of a previous optimization, which has proved to generate a matrix comparable with those obtained with standard decellularization methods both in terms of residual mechanical properties ${ }^{28}$ and at the immunohistochemical level. $^{29}$ Intact not decellularized human reticular dermis coming from the same donors was used as a control. Both the native human reticular dermis (Control) and HADM used for these experiments were preserved at 85 per cent glycerol in a $4^{\circ} \mathrm{C}$ refrigerator. The specimens were obtained by cutting out $30 \times 30 \mathrm{~mm}$ squares using a custom-made die cutter. A maximum of four specimens from each strip was obtained, except for the not-decellularized strip (Control) coming from donor $B$, whose small size have enabled the obtaining of two specimens. Therefore, 22 specimens were tested in total.
Figure 2: Strain distribution along the $x$ direction ( $\varepsilon x x), y$ direction ( $\varepsilon y y)$ and equivalent Von Mises Strain distribution ( $(\varepsilon e q)$ in the intermediate $(t=30 s)$ and final instant $(t=60 s)$

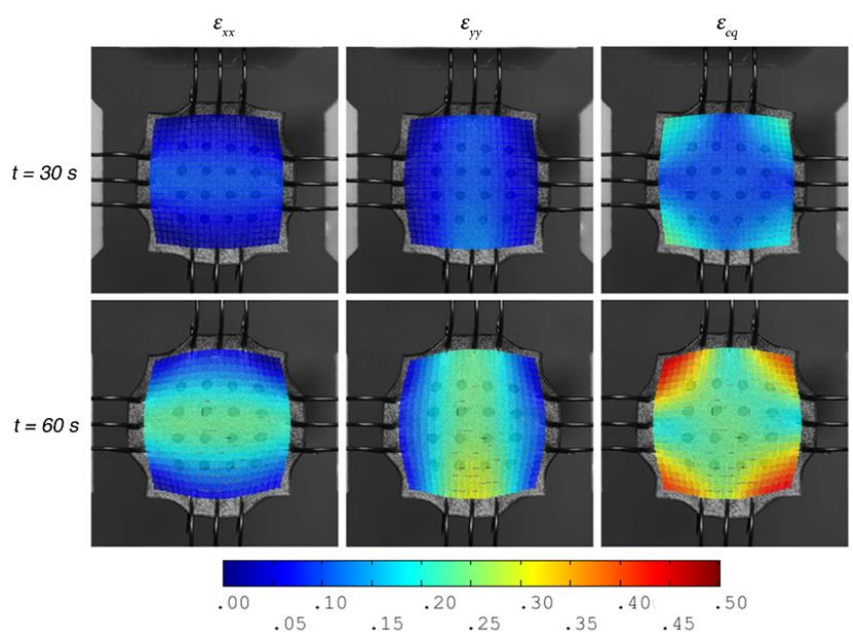

The cutting orientation with respect to the sampling direction has been indicated by means of stitches sewed at the northwest vertex of the square. This numbering is needed in order preserve the original orientation and location during the measurement and washing procedures. Before testing, the dermis grafts were washed to remove all the glycerol, dipping them sequentially in three different beakers filled with abundant saline solution 0.9 per cent at $+37^{\circ} \mathrm{C}$ for more than three minutes each. ${ }^{28}$ The specimens thickness at rest was then obtained by means of photogrammetry (full-frame digital camera - Canon EOS 5D Mark II - with an autofocus lens for macro photography Canon EF $100 \mathrm{~mm}$ f/2.8 Macro USM) as an average of five measures evaluated using the image analysis software ImageJ (National Institutes of Health, Bethesda, Maryland, U.S.). An average thickness equal to $2.43 \pm 0.46 \mathrm{~m} \mathrm{~mm}$ (average \pm std) was obtained.

\section{Biaxial mechanical tests}

Specimens were mounted on the biaxial testing device and a $4 \times 4$ matrix of markers was then dawn on the visible surface with an alcohol-based permanent black ink (Figure $3)$. The test speed was set to $0.16 \mathrm{~mm} / \mathrm{s}$ and the test was stopped at the achievement of a $10 \mathrm{~mm}$ displacement on each axis. The previously described digital camera was used to capture images of the specimen during the test with a 1 $\mathrm{Hz}$ frame rate.

\section{Data elaboration}

The loads were acquired by two force transducers (Honeywell Sensotec Sensors Model 31 Mid) positioned along the two loading directions, while the marker 
displacements were measured optically using the photogrammetry set-up. The true stresses were then calculated by dividing the load cell readings by the crosssectional area of the specimen, which was taken as the product of the gauge length in the two orthogonal directions $\left(L_{M L}, L_{C C}\right)$ and the thickness of the specimen over time.

The gauge lengths were evaluated through the ImageJ image analysis software as an average of three measures of the distance between the hooks (Figure 3), orthogonally to the loading direction (eqns (1) - (2)).

Figure 3: Parameters extraction for stress and strain evaluation. The white square borders the gauge lengths in the mediolateral (LML) and in the craniocaudal (LCC) directions. The black squares enclose the four central markers in the undeformed and the deformed state. Lengths used for the strain evaluations are highlighted

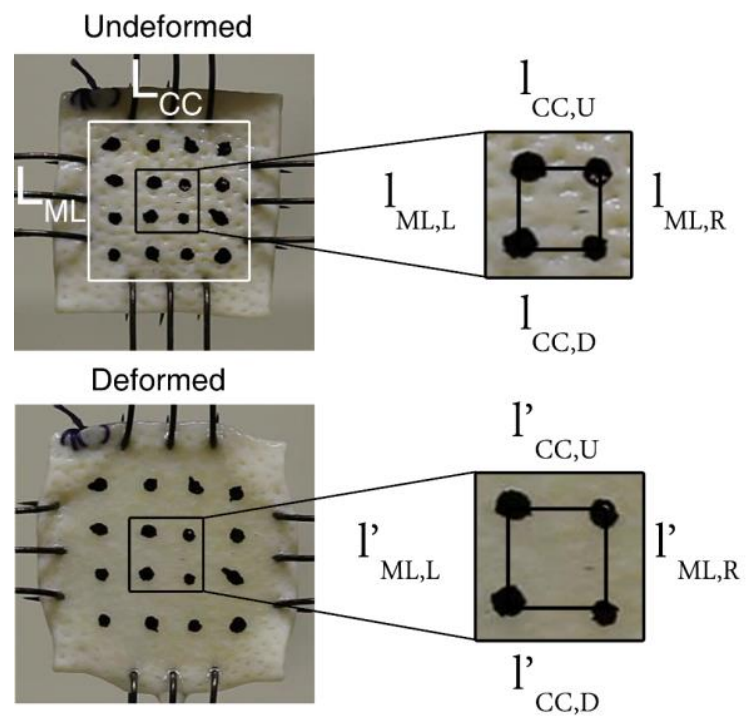

$\sigma_{M L}(t)=\frac{F_{M L}(t)}{L_{C C}(t) \cdot s(t)}$

$\sigma_{C C}(t)=\frac{F_{C C}(t)}{L_{M L}(t) \cdot s(t)}$

The strain of the specimen along the two loading directions was then determined from the distance increase between the central markers of the specimen in the horizontal and vertical directions (eqns (3) - (4)). Markers centroids and trajectories were identified by a segmentation process performed in the Matlab environment.

$\varepsilon_{M L}(t)=\operatorname{mean}\left(\frac{l_{M L, L}^{\prime}(t)-l_{M L, L}}{l_{M L, L}} ; \frac{l_{M L, R}^{\prime}(t)-l_{M L, R}}{l_{M L, R}}\right)$
$\varepsilon_{C C}(t)=\operatorname{mean}\left(\frac{l_{C C, U}^{\prime}(t)-l_{C C, U}}{l_{C C, U}} ; \frac{l_{C C, D}^{\prime}(t)-l_{C C, D}}{l_{C C, D}}\right)$

Where $L M L(t)$ and $L C C(t)$ are the mean distances between the hooks insertion points in the vertical and in the horizontal direction respectively, $\mathrm{FML}(\mathrm{t})$ and $\mathrm{FCC}(\mathrm{t})$ are the vertical and horizontal load cells outputs and $s(t)$ is the specimen thickness over time, evaluated knowing the LML and LCC arrays and assuming an isochoric condition.

All parameters meanings are depicted in Figure 3. From the experimental data obtained, the slope of the toe region was evaluated from 0 to a 10 per cent of strain, as an indication of the matrix deformability at low strains. Showing the stress-strain curves an almost exponential trend, their slope at higher strains was evaluated in a semi-log plane. Curves were thus fitted in the Matlab environment with a singleterm exponential model (eqn (5)), being the growth constant $b$ the slope of the stress-strain curve in the semilog plane, or, in other words, the fibre rearrangement rate with respect to the experienced strain (Figure 4).

$\sigma(\varepsilon)=a e^{b \varepsilon}$

A statistical analysis of the experimental results was carried out using a multivariate analysis of variance (Two Way ANOVA). The two analysed factors were the "donor" (three levels: A, B and C) and the "direction" (two levels: mediolateral and craniocaudal). The significance level were set to $p<0.05$ for all tests.

Figure 4: Exponential fitting of mediolateral and craniocaudal curves in a representative specimen (Left), and plot of the same curves in a semi-log plane, with indication of the $b$ slope (Right)
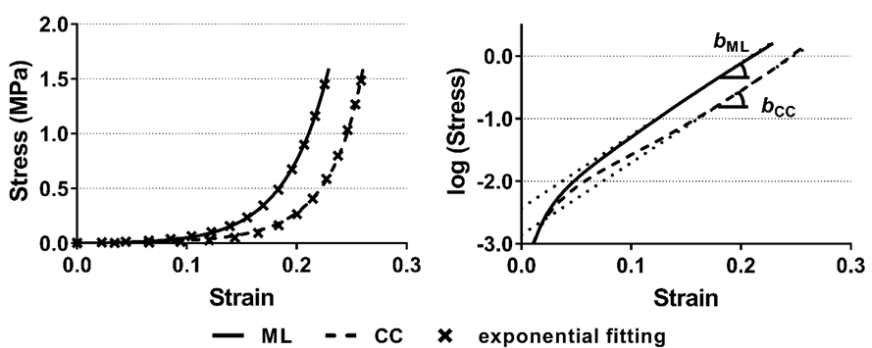

\section{Results}

A representative set of results of the biaxial tensile tests can be found in Figure 5. It shows the stress-strain curves of four Control specimens (native human reticular dermis) coming from the same donor (A). In some cases, test was not completed with success due to human errors during the experiment, resulting in a single result for medio-lateral direction of the not-treated specimens coming from donor 
B. The slope of the toe region in a physiological range of strains (0-10 per cent) was evaluated from each experimental curve. ${ }^{32}$ Results are represented in Figure 6 and in Table 1.

\section{Discussion}

Results underline the anisotropic behaviour of dermis, since the mechanical response in one direction appears, most of the times, stiffer that the one in the orthogonal direction. In particular, the overall result for the biaxial data in the medio-lateral direction was higher than the cranio-caudal direction. This can be explained by the predominance of collagen fibres aligned along the medio-lateral direction in the dermis tissue taken from the lower back, which is in accordance with Langer findings ${ }^{33}$ and later works. ${ }^{12,34}$ Similar findings were described by Lanir et al. ${ }^{25}$ regarding the rabbit skin. They found a stiffer behaviour of the tissue along the body width (medio-lateral direction) then along the body length, when biaxially solicited. The high material anisotropy causes a not homogenous strain distribution along the specimen, also affecting its central ROI, in which the characteristic curve of the material is evaluated.

\section{Figure 5: A representative stress-strain set of curves} (Control results obtained from donor A). Grey plots show a magnification of the curves toe-region from 0 to 5 per cent of strain
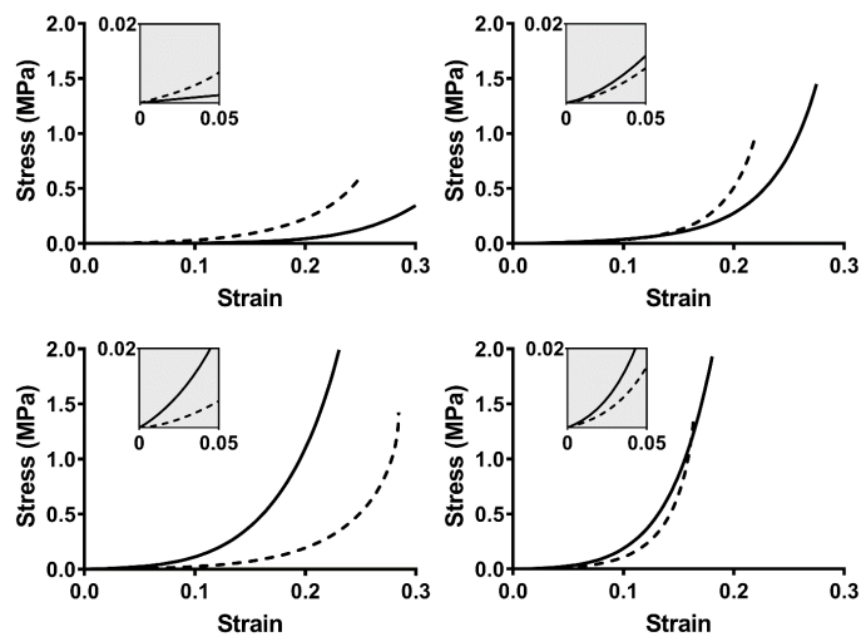

$-\mathrm{ML}--\mathrm{CC}$
Figure 6: Average values \pm the range of variation of: elastic modulus evaluated at 10 per cent of strain (Left) and slope of the stress-strain curve in the semi-log plane (b) (Right) for the medio-lateral and the cranio-caudal directions. A, B and $\mathrm{C}$ are the three tested donors
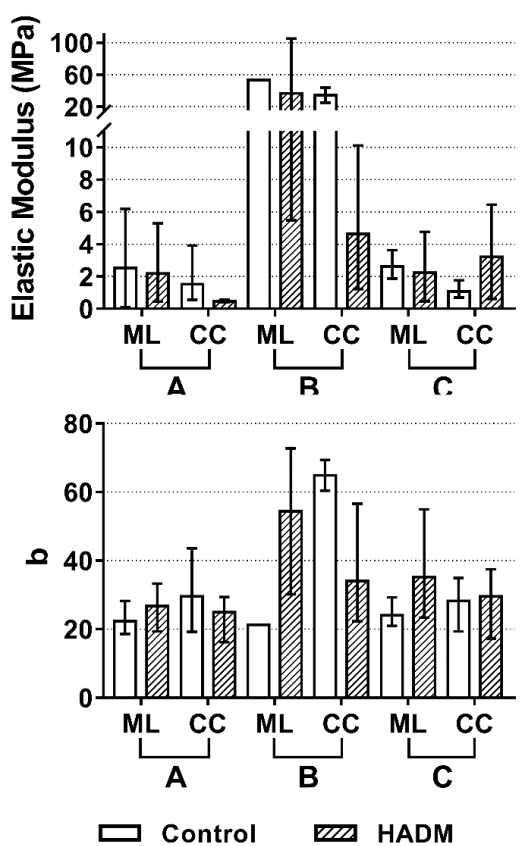

Previous studies reported results regarding the percentage of the area which can be considered characterized by a uniform strain distribution. For instance, Humphrey et al. ${ }^{35}$ reported a uniform strain distribution within the central 4 per cent of the specimen area, while Billiar et al. ${ }^{27}$ found a uniformity of strains in the central 25 per cent of the specimen area, but specifying that a homogeneous isotropic material loaded at four discrete locations per side was tested. For an anisotropic tissue, the stress-strain distribution would be less uniform, thus a smaller percentage should be considered, even though direct experimental evidence is needed. Nevertheless, Oomens et al. ${ }^{36}$ claims that a homogeneous strain cannot be obtained because of the inhomogeneous materials properties and the challenging (sometimes impossible) manufacturing of sample. Anyhow, in this work about the 5 per cent of the gauge area (from hooks to hooks) was considered for the stress-strain curves extraction. 
Table 1: Elastic modulus of the toe-region and slope of the stress-strain curve in the semi-log plane (b) for the mediolateral (ML) and the cranio-caudal (CC) directions of donors A, B and C

\begin{tabular}{|c|c|c|c|c|c|}
\hline \multirow{4}{*}{ Donor } & \multirow{3}{*}{ Dir. } & \multicolumn{2}{|c|}{$\begin{array}{c}\text { Elastic modulus } \\
\text { (MPa) }\end{array}$} & \multicolumn{2}{c|}{ b } \\
& & (average \pm SD) & \multicolumn{2}{c|}{ (average \pm SD) } \\
\cline { 3 - 6 } & & Control & HADM & Control & HADM \\
\hline \multirow{4}{*}{ A } & \multirow{2}{*}{ ML } & 2.53 & 2.18 & 22.38 & $26.77 \pm 5.8$ \\
& & \pm 2.75 & \pm 2.13 & \pm 4.14 & 1 \\
\cline { 2 - 6 } & \multirow{2}{*}{ CC } & 1.52 & 0.46 & 29.58 & $24.97 \pm 6.1$ \\
& & \pm 1.60 & \pm 0.07 & \pm 10.42 & 6 \\
\hline \multirow{4}{*}{ B } & \multirow{2}{*}{ ML } & 52.99 & 36.64 & 21.33 & $54.34 \pm 18$. \\
& & $\pm n . a$. & \pm 47.09 & $\pm n . a$. & 83 \\
\cline { 2 - 6 } & \multirow{2}{*}{ CC } & 34.31 & 4.64 & 64.92 & $34.02 \pm 15$. \\
& & \pm 13.40 & \pm 4.05 & \pm 6.32 & 58 \\
\hline \multirow{4}{*}{ C } & \multirow{2}{*}{ ML } & 2.62 & 2.23 & 24.00 & $35.21 \pm 13$. \\
& & \pm 0.80 & \pm 1.82 & \pm 3.63 & 72 \\
\cline { 2 - 6 } & \multirow{2}{*}{ CC } & 1.06 & 3.22 & 28.22 & $29.56 \pm 9.5$ \\
& & \pm 0.49 & \pm 2.44 & \pm 7.23 & 3 \\
\hline
\end{tabular}

The higher stiffness of the tissue in the medio-lateral direction (along Langer lines) is here confirmed ( $p=0.0055)$, with standard deviations relatively limited. Only exception the donor $B$, which shows in general higher average values of elastic modulus and, in the decellularized tissues, shows high standard deviations along the medio-lateral direction $(3.976 \pm 4.079 \mathrm{MPa})$. The mean elastic modulus for the medio-lateral direction resulted from 35 per cent to 87 per cent higher than the cranio-caudal one, except for the decellularized tissue coming from donor $\mathrm{C}$ which shows a cranio-caudal elastic modulus 40 per cent higher than the medio-lateral one. In the lower back, from where the dermis employed in this study was collected, the Langer's lines are oriented mainly along the mediolateral direction and start to curve along the orthogonal direction approaching the hips. ${ }^{33}$ Inaccuracies in tissue harvesting can therefore lead to errors in the orientation of the specimen during mechanical tests, causing the discrepancy found in donor C. Interesting to note is that coupled results emerge from the comparison between the elastic modulus in the toe-region and the parameter $b$, which describes the fibres rearrangement rate along the two loading directions at high strains. Indeed, when the tissue responds stiffer at lower strains, it shows lower rearrangement velocities at higher strains, revealing a lower propensity to realignment, namely, a stiffer response to imposed deformations.

Comparing the paired native and decellularized groups of specimens, the reduction of the mechanical properties of the treated dermis with respect to the native tissue is here again confirmed for all donors, and considering both directions. $^{28}$

Surgical procedures begin and end with incisions, and the incisions planning is a mandatory step when a good outcome is desired in terms of scars and tension distribution in the scar proximity. ${ }^{36}$ The incision planning is intimately related to the Langer's lines orientation, because incisions made parallel to Langer's lines may heal better and produce less scarring than incisions made across Langer's lines. ${ }^{37,38}$ This consideration can also be shifted in cases which do not directly involve aesthetics, such as soft tissues reconstructive surgeries, were a biological graft is needed. When placed in a body part where the substitute will bear a physiological and anisotropic distribution of stress, the orientation of the graft should be consistent with the surrounding tissue. Therefore, not only the stiffer direction of the host tissue should be known, but also the substitute one and, ideally, an equi-biaxial test performed on a small square specimen excerpts from the graft could provide this indication. To provide a comprehensive characterization of the tissue, in the future we will investigate the mechanical behaviour and the architectural heterogeneity of the HADM at the microscale $e^{39,40}$ to understand its influence on the behaviour of the tissue at the macroscale.

\section{Conclusion}

The biaxial mechanical behaviour of native dermis and HADM was measured exploiting a purpose-made biaxial conversion device. Stress-strain curves were evaluated from the loads acquired by two load cells positioned along two orthogonal axes and the optical measure of the displacements of four markers located in the central area of the specimen. Comparisons among the native and decellularized tissues demonstrated the loss of mechanical strength caused by the decellularization treatment. Moreover, the specimens resulted, on average, less extensible in the medio-lateral direction (namely, along the Langer lines) then in the cranio-caudal direction, confirming the correlation of dermis mechanical response with collagen fibres orientation with respect to the loading direction.

\section{References}

1. Shi L, Wang J, Yang C, et al. Application of acellular dermal matrix in reconstruction of oral mucosal defects in 36 Cases. J Oral Maxillofac Surg. 2012;70(11):586-91.

2. Duncan D. Correction of implant rippling using allograft dermis. Aesthet Surg J. 2001;21(1):81-4.

3. Breuing K, Warren S. Immediate bilateral breast reconstruction with implants and inferolateral AlloDerm slings. Ann Plast Surg. 2005;55(3): 232-9. 
4. Garvey P, Martinez R, Baumann D, et al. Outcomes of abdominal wall reconstruction with acellular dermal matrix are not affected by wound contamination. J Am Coll Surg. 2014;219(5):853-64.

5. Barber $F$, Herbert $M$, Boothby $M$. Ultimate tensile failure loads of a human dermal allograft rotator cuff augmentation. Arthroscopy. 2008;24(1):20-4.

6. Burkhead W, Schiffern S, Krishnan S. Use of Graft Jacket as an Augmentation for Massive Rotator Cuff Tears. Seminars in Arthroplasty. 2007;18(1):11-8.

7. Snyder S, Bond J. Technique for arthroscopic replacement of severely damaged rotator cuff using "GraftJacket" allograft. Operative Techniques in Sports Medicine. 2007;15(2):86-94.

8. Wong I, Burns J, Snyder S. Arthroscopic GraftJacket repair of rotator cuff tears. J Shoulder Elbow Surg. 2010;19(2S):104-9.

9. Lee D. Achilles Tendon repair with acellular tissue graft augmentation in neglected ruptures. J Foot Ankle Surg. 2007;46(6):451-5.

10. Barber F, Herbert M, Coons D. Tendon augmentation grafts: Biomechanical failure loads and failure patterns. Arthroscopy. 2006;22(5):534-8.

11. Moore M, Samsell B, Wallis G, et al. Decellularization of human dermis using non-denaturing anionic detergent and endonuclease: a review. Cell Tissue Bank. 2015;16(2):249-259.

12. Nì Annaidh $A$, Bruyère $K$, Destrade $M$, et al. Characterization of the anisotropic mechanical properties of excised human skin. J Mech Behav Biomed Mater. 2012a;5:138-48.

13. Aldieri A, Terzini M, Bignardi $C$, et al. Implementation and validation of constitutive relations for human dermis mechanical response. Med Biol Eng Comput. 2018

14. Waldman S, Lee J. Boundary conditions during biaxial testing of planar connective tissues. Part 1: Dynamic Behavior. J Mater Sci Mater Med. 2002;13(10):933-8.

15. Skulborstad A, Swartz S, Goulbourne N. Biaxial mechanical characterization of bat wing skin. Bioinspir Biomim. 2015;10(3):1-16.

16. Grashow J, Yoganathan A, Sacks M. Biaixal stress-stretch behavior of the mitral valve anterior leaflet at physiologic strain rates. Ann Biomed Eng. 2006;34(2):315-25.

17. Zemánek $M$, Burša J, Děták $M$. Biaxial tension tests with soft tissues of arterial wall. Eng Mech. 2009;16(1):3-11.

18. Prendergast $P$, Lally $C$, Daly $S$, et al. Analysis of prolapse in cardiovascular stents: A constitutive equation for vascular tissue and finite-element modelling. J Biomech Eng. 2003;125(5):692-9.
19. Zanetti EM, Perrini M, Bignardi C, et al. Bladder tissue passive response to monotonic and cyclic loading. Biorheology. 2012;49(1):49-63.

20. Lally C, Reid A, Prendergast P. Elastic behavior of porcine coronary artery tissue under uniaxial and equibiaxial tension. Ann Biomed Eng. 2004;32(10):1355-64.

21. Kahlon A, Hurtig M, Gordon K. Regional and depth variability of porcine meniscal mechanical properties through biaxial testing. J Mech Behav Biomed Mater. 2015;41:108-14.

22. Zanetti EM, Bignardi C. Structural analysis of skeletal body elements: numerical and experimental methods. in Biomechanical systems technology (edited by Cornelius $\mathrm{T}$ Leondes (University of California, Los Angeles, USA), World Scientific Publishing. 2009;3:185-225.

23. Sacks M. Biaxial mechanical evaluation of planar biological materials. J Elast. 2001;61:199-246.

24. Lanir Y, Fung Y. Two-dimensional mechanical properties of rabbit skin-l. Experimental system. J Biomech. $1974^{\circ} ; 7(1): 29-34$.

25. Lanir Y, Fung Y. Two-dimensional mechanical properties of rabbit skin-II. Experimental results. J Biomech. 1974b;7(2):171-82.

26. Tong $P$, Fung $Y$. The stress strain relationship for the skin. J Biomech. 1976;9(10):649-657.

27. Billiar K, Sacks M. Biaxial Mechanical Properties of the Natural and Glutaraldehyde Treated Aortic Valve Cusp Part I: Experimental Results. J Biomech Eng. 2000;122:23-30.

28. Terzini M, Bignardi C, Castagnoli C, et al. Dermis mechanical behaviour after different cell removal treatments. Med Eng Phys. 2016;38(9):862-9.

29. Ferrando PM, Balmativola D, Cambieri I, et al. Glycerolized reticular dermis as a new human acellular dermal matrix: An exploratory study. PLoS One. 2016;11(2):e0149124.

30. Terzini M, Bignardi C, Castagnoli C, et al. Ex vivo dermis mechanical behavior in relation to decellularization treatment length. Open Biomed Eng J. 2016;10:34-42.

31.Jones E. Documentation for Matlab-base DIC code, Illinois: Copyright (c) 2013, Elizabeth Jones, 2013.

32. Manschot J, Brakkee A. The measurement and modelling of the mechanical properties of human skin in vivo -- I. The measurement. J Biomech. 1986;19(7):511-5.

33. Langer K. On the anatomy and physiology of the skin. I. The cleavability of the cutis. $\mathrm{Br} J$ Plast Surg. 1978;31(1):3-8.

34. Yoder J, Elliott D. Nonlinear and anisotropic tensile properties of graft materials used in soft tissue applications. Clin Biomech (Bristol, Avon). 2010;25(4):378-82. 
35. Humphrey JVDVR. Quantification of strains in biaxially tested soft tissues. J Biomech. 1987;20(1):59-65.

36. Oomens CWJ, van Ratingen MR, Janssen JD, et al. A numerical-experimental method for a mechanical characterization of biological materials. J Biomech. 1993;26(4/5):617-21.

37. Waldorf J, Perdikis G, Terkonda S. Planning incisions. Operative Techniques in General Surgery. 2002;4(5):199-206.

38. Physiology, "Lines of Cleavage and Surgery," July 21th 2015. Available:

https://www.boundless.com/physiology/textbooks/bou ndless-anatomy-and-physiology-textbook/theintegumentary-system-5/skin-diseases-disorders-injuryand-clinical-cases-69/lines-of-cleavage-and-surgery-424184/. [Accessed 20 March 2016].

39. Massai D, Pennella F, Gentile $P$, et al. Image-based three-dimensional analysis to characterize the texture of porous scaffolds. Biomed Res Int. 2014;2014:161437.

40. Bignardi C, Petraroli M, Pugno NM. Nanoindentation on conch shells of Gastropoda and Bivalvia molluscs reveal anisotropic evolution against external attacks. J Nanosci Nanotechnol. 2010;10(10):6453-60.

\section{PEER REVIEW}

Not commissioned. Externally peer reviewed.

\section{CONFLICTS OF INTEREST}

The authors declare that they have no competing interests.

\section{FUNDING}

No specific funding has been used for this research. 